Provided for non-commercial research and education use. Not for reproduction, distribution or commercial use.

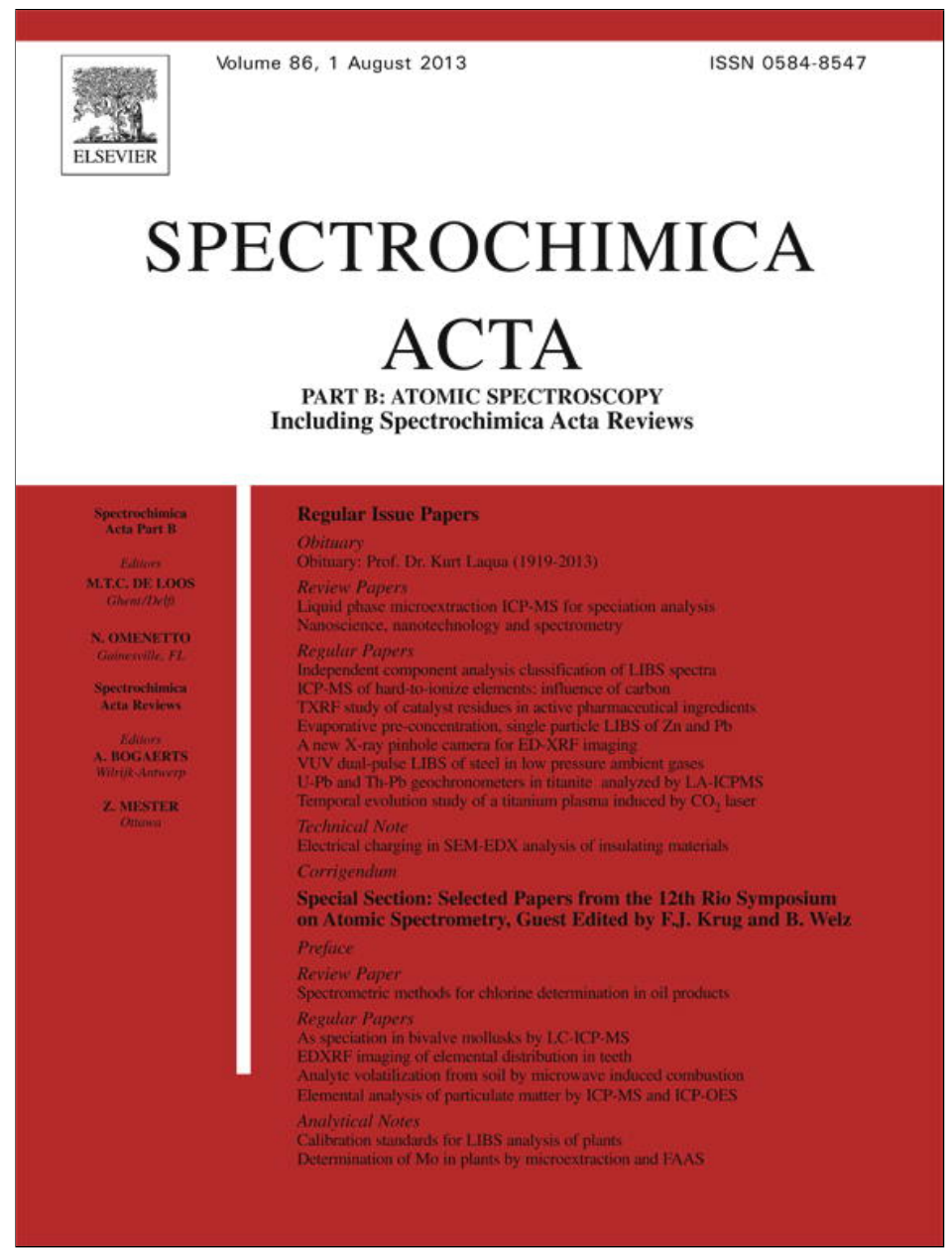

This article appeared in a journal published by Elsevier. The attached copy is furnished to the author for internal non-commercial research and education use, including for instruction at the authors institution and sharing with colleagues.

Other uses, including reproduction and distribution, or selling or licensing copies, or posting to personal, institutional or third party websites are prohibited.

In most cases authors are permitted to post their version of the article (e.g. in Word or Tex form) to their personal website or institutional repository. Authors requiring further information regarding Elsevier's archiving and manuscript policies are encouraged to visit:

http://www.elsevier.com/authorsrights 
Review

\title{
Nanoscience, nanotechnology and spectrometry
}

\author{
Freddy C. Adams ${ }^{\text {a }}$, Carlo Barbante ${ }^{\text {b,c,* }}$ \\ a Department of Chemistry, University of Antwerp, B-2610 Wilrijk, Belgium \\ b Institute for the Dynamics of Environmental Processes - CNR, Venice, Italy \\ ' Department of Environmental Sciences, Informatics and Statistics, Ca' Foscari University, Venice, Italy
}

\section{A R T I C L E I N F O}

\section{Article history:}

Received 26 February 2013

Accepted 16 April 2013

Available online 10 May 2013

\section{Keywords:}

Nanoscience

Nanotechnology

Nanomaterial

Chemical analysis

Nanoanalysis

\begin{abstract}
A B S T R A C T
Nanoscience has outgrown its infancy, and nanotechnology has found important applications in our daily life with many more to come. Although the central concepts of the nano world, namely the changes of particular physical properties on the length scale of individual atoms and molecules, have been known and developed for quite some time already, experimental advances since the 1980s and recognition of the potential of nanomaterials led to a genuine breakthrough of the inherently multidisciplinary nanoscience field. Analytical nanoscience and nanotechnology and especially the use of micro and nano electro mechanical systems, of the quantum dots and of mass spectrometry, currently provide one of the most promising avenues for developments in analytical science, derived from their two main fields of action, namely (a) the analysis of nano-structured materials and (b) their use as new tools for analysis. An overview is given of recent developments and trends in the field, highlighting the importance and point out future directions, while also touching drawbacks, such as emerging concerns about health and environmental issues.
\end{abstract}

(c) 2013 Elsevier B.V. All rights reserved.

\section{Contents}

1. Introduction ... . . . . . . . . . . . . . . . . . . . . . . . . . . . . . . . . . . . . . 4

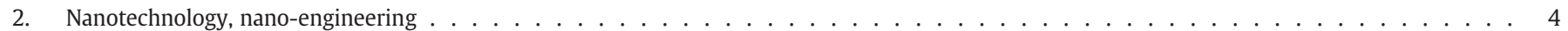

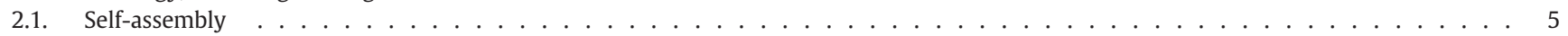

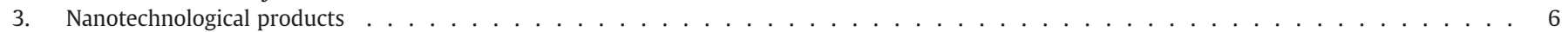

3.1. Two examples . . . . . . . . . . . . . . . . . . . . . . . . . . . . . . . . . . . . . . 6

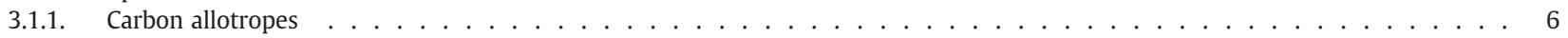

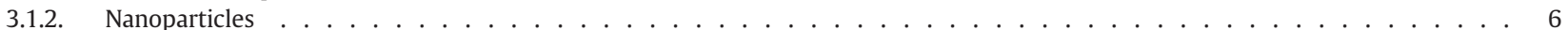

4. Nanoscience and analytical chemistry . . . . . . . . . . . . . . . . . . . . . . . . . . . . . . . . . . . . . . . . . . . 7

4.1. Analytical chemistry . . . . . . . . . . . . . . . . . . . . . . . . . . 7

4.1.1. Biomimetics . . . . . . . . . . . . . . . . . . . . . . . . . . . . . 7

4.2. Chemical analysis and nanoscience/technology . . . . . . . . . . . . . . . . . . . . . . . . . . 8

4.2.1. Imaging and analysis . . . . . . . . . . . . . . . . . . . . . . . . . . . . . . . . . . . . 8

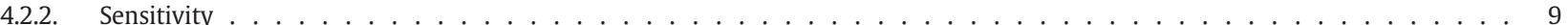

4.2.3. Abbe-Thomson diffraction limit . . . . . . . . . . . . . . . . . . . . . . . . . . . . . . . 9

4.3. Nanomaterials as analytical tools ... . . . . . . . . . . . . . . . . . . . . . . . . . . . 9

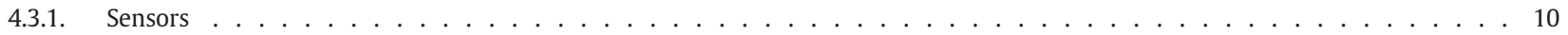

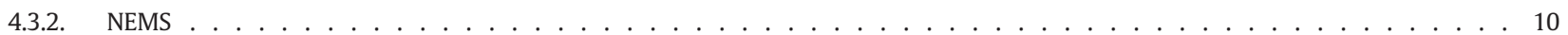

4.3.3. Nanoparticles, quantum dots and spectroscopy . . . . . . . . . . . . . . . . . . . . . . . . . . . . . 11

4.3.4. Mass spectrometry . . . . . . . . . . . . . . . . . . . . . . . . . . . . . . . . . . . . . . . . . . . . . 11

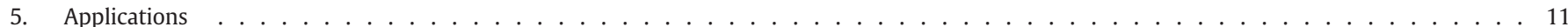

5.1. Applications in biology and medicine . . . . . . . . . . . . . . . . . . . . . . . . . . . . . . . . 12

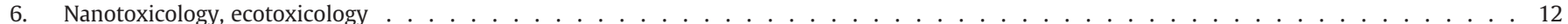

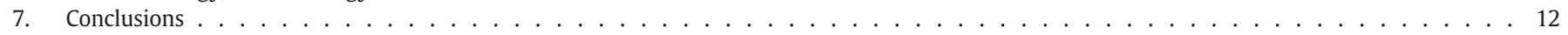

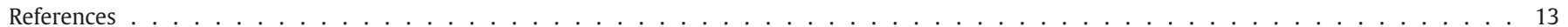

\footnotetext{
* Corresponding author at: Department of Environmental Sciences, Informatics and Statistics, Ca' Foscari University, Venice, Italy.

E-mail address: barbante@unive.it (C. Barbante).
} 


\section{Introduction}

Nano-science and nanotechnology are based on the manipulation of individual atoms and molecules to produce materials from them for applications well below the sub-microscopic level. They involve physical, chemical and biological knowledge at scales ranging between individual atoms and molecules below the nanometer, up to ca $100 \mathrm{~nm}$. The subject also concerns the integration of the resulting structures into larger systems. Many definitions of nanotechnology refer to dimensions: according to the National Nanotechnology Initiative (NNI) in the United States, for instance, "nanotechnology is the understanding and control of matter at dimensions between approximately 1 and 100 nanometres, where unique phenomena enable novel applications". Often only one or two dimensions are in the nanoregime, as in quantum wells and nanowires but sometimes all three dimensions are nanoscale, as in quantum dots and nanocrystals. A challenge is to make every dimension as small as possible, as in nanoelectronics, but other times the aim is to make at least one dimension as large as possible, as in carbon nanotubes.

A material consisting of a nanolayer surface, as for instance ultrathin coatings, is not necessarily considered as such as a nano-material. If only one of the dimensions is restricted, we have a layered shape or 2-D material; if two dimensions are limited in size, we will have a wired or 1-D material; if all dimensions are in the range of a few nanometres we have 0-D materials.

Many aspects of nanotechnology are based on the fact that the nanoscale world is different from the macroscopic world that we are so well accustomed to deal with in life and in applications. At the micron size level and above, materials have bulk - conventional properties that obey the laws of classical science, while submicroscopic objects - mesoscopic between $1 \mu \mathrm{m}$ and $100 \mathrm{~nm}$ and nanoscopic below this size range - have properties that are affected by fluctuations around the average and become subject to the strange and unpredictable laws of quantum mechanics. In this way, many exciting new tools and functionalities are opening up in this new technological field. And this leads also to a plethora of new economic challenges and opportunities in the scientific realm too.

As length scales of materials decrease, surface-area effects become extremely important and quantum effects appear that lead to profound changes in the properties of materials and devices. One effect of the small size of the materials is the increasing percentage of atoms that are situated on the surface of the objects. There is a famous quote from Wolfgang Pauli that was expressed long ago, it says:

"God made the bulk, the surface was invented by the Devil".

With this logic, in nano-sized materials the Devil's realm has been extended enormously. In nanoscience and technology surface physics and chemistry start dominating the materials properties and this must be duly taken into account. The large percentage of atoms on the surface for small entities, and the reactivity that this gives rise to, concerns one of the principal factors that differentiate properties of nanostructures from those of the bulk material.

One important result of the high surface area per unit mass is the reactivity of nano-size materials. If, for instance, the surface of nanoparticles is not protected through surface passivation, interactions between the particles will readily occur. Thus, in nanotechnology common concepts of chemistry lose their validity: gold nanoparticles are very reactive while argon clusters of up to several thousand atoms are stable enough to be used as projectiles in diverse scientific and technological processes.

The property changes resulting from the size effects lead to both advantages and drawbacks. For example, on one side, there is the ability of quantum particles to tunnel through barriers that repel classical particles; this, for instance, underpins the operation of the scanning tunnelling microscope, a revolutionary new way of the observation of nature.
On the other side, the same tunnelling effect can be a source of serious problems in ultra small-scale nano-electronic devices.

One other aspect to consider is that as structures enter the nanoscale regime, the van der Waals force increases. For example, it is this force that clamps graphene samples to substrates, and also holds together individual graphene sheets in multi-layer samples.

Microstructures, electronics, nanotechnology are large fields of R\&D. Increasingly they are now growing together. Recently, newly commercialized products indicate the innovative potential and future applications. Essential for future developments is to keep track of the composition and the structure. For that, the pursuit of chemical analysis at extremely small size levels is necessary.

We cannot go here into any detail on the vastly expanding research on nanotechnology and nanoscience. A comprehensive handbook of Bushan [1], for instance, is almost 2000 pages in its last edition.

There is a wide variety of analytical techniques that may provide elemental and molecular information with high spatial resolution and that are currently used for atomic scale observation, determination of structure and for the quantification of the chemical composition. Analytical techniques in the field of nano-analyses benefitted of the enormous progress made in the digital realm, which allowed the high speed of signal acquisition, and on the other side, in the development in nano-sized materials and tools which are mostly used as analytical probes. The synergy created by this combination allowed the development of sophisticated analytical imaging tools that combine high sensitivity with unrivalled spatial discrimination potential.

\section{Nanotechnology, nano-engineering}

The physicochemical properties of nanomaterials significantly depend on their three-dimensional morphologies - sizes, shapes and surface topography - the surrounding media, and their arrangement in space. The correlation of these parameters with the relevant physical and chemical properties is a fundamental requirement for the discovery of novel properties and applications - as well as for advancing the fundamental and practical knowledge required for the design and fabrication of new materials.

Nano-engineering concepts are crudely defined in Fig. 1. In general, there are three ways to engineer complex entities of reduced dimensions based on bottom-up and top-down approaches or self-assembly. The first consists in the manipulation of atoms into the desired structures. Such bottom-up fabrication forms device structures directly from mechanisms of material growth such as atomic layer deposition methods [2]. The objectives are to achieve:

- The ultimate precision: almost every atom in exactly the right place in space.

- To make complex and molecularly intricate structures as easily and inexpensively as the materials we are accustomed to work with now.

- Reduce manufacturing costs to little more than the cost of the required raw materials and energy.

The evolution that was realized in the bottom-up approach over the last 20 years is shown on the left. A landmark on this approach was the well-known IBM image constructed in 1989 from 35 individual xenon atoms on a nickel surface using a scanning tunnelling microscope (STM) for positioning the atoms. The STM was invented to image surfaces at the atomic scale, but has been used as a tool with which atoms and molecules can be manipulated to create structures. The tunnelling current can be used to selectively break chemical bonds or to induce chemical associations. Such an atom by atom handling approach enables almost any structure to be built, but is too slow for bulk fabrication of copies in mass production.

The top-bottom strategies for manufacturing nano-sized materials consists in downscaling conventional methods, such as in the procedures used in the semiconductor industry's International Technology 


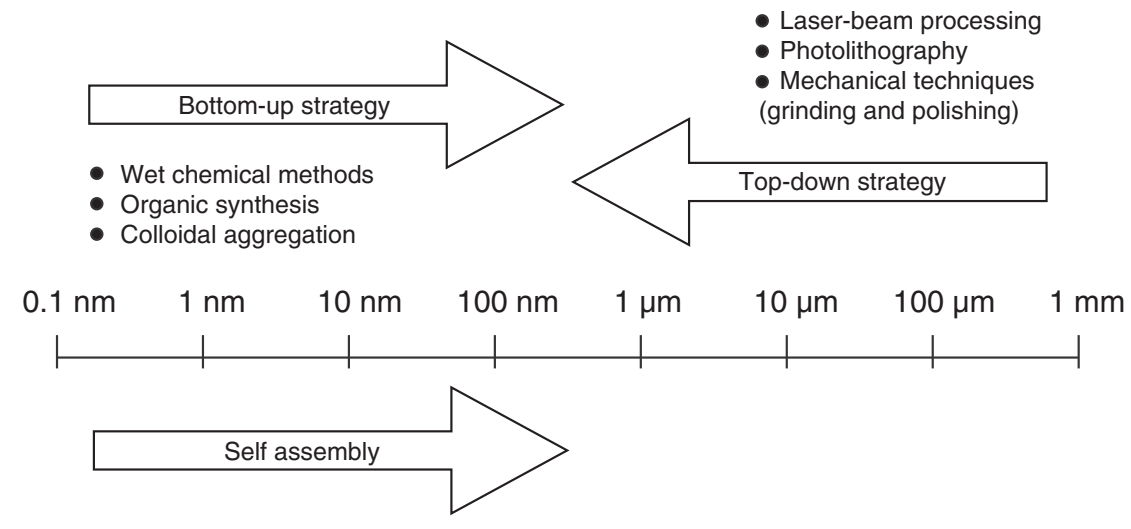

Fig. 1. Top-down and bottom-up nanotechnology. Adapted from reference [45].

Roadmap for Semiconductors. Buck and Shoulders first described a topdown nanofabrication and nano-engineering procedure by which nanostructured electronics might be written by electron beams in 1958 and provided a vision on nanofabrication and nano-engineering as it developed since then [3,4]. In what follows there is a quote from this prophetic paper:

"The day is rapidly drawing near when digital computers will no longer be made by assembling thousands of individually manufactured parts into plug-in assemblies and then completing their interconnection with back-panel wiring. An alternative to this method is one in which an entire computer or a large part of a computer is made in a single process. Vacuum deposition of electrodes onto blocks of pure silicon or germanium and the subsequent diffusion of the electrode material into the block to form junctions is a most promising method. The successful development of this method would allow large numbers of transistors and all of their interconnecting wiring to be made in one operation".

At present, top-down nanofabrication produces nanometer scale devices from bulk materials by lithography techniques, which include photo-lithography and electron beam lithography. Top-down nanofabrication and nano-engineering procedures were used from the late 1950s on for nanostructured electronics and they are still relevant for nano-electronics at present. Standard optical and electron beam lithography techniques struggle to produce mask feature sizes below $30 \mathrm{~nm}$, but scanning probes provide a resolution of $15 \mathrm{~nm}$ at a speed comparable to electron beam lithography. Nano-patterning enables other applications, that are increasingly adopted in nanotechnology and improvements directly gives rise to new applications, including those in analytical chemistry (for instance in sensors), accelerating the rate at which new applications can be developed in the future. The evolution that was realized in the bottom-up approach over the last 20 years is shown in Fig. 2. It gives an example of the evolution of the state-of-the-art of micromanipulation in 1989 (with the bottom-up approach) and 2009 (top-down approach) [5].

\subsection{Self-assembly}

The established pick-and-place assembly techniques or the topbottom approach may be unsuitable in many situations and this brings us a third approach, self-assembly, the third possibility to engineer complex nano-size objects. This follows from the science of supra-molecular chemistry, which involves the ability of atoms and molecules to react spontaneously to form complex structures as a result of their physical and chemical interactions. Self-assembly is based on engineering the interactions between particles by chemically functionalizing their surfaces, so that they self-assemble to form the desired structure. The principles of supramolecular chemistry were pioneered by e.g. the Nobel laureate Jean-Marie Lehn and others in the 1970s and 1980s. Supramolecular chemistry shows how molecules (a prominent example is DNA) are imbued with instructions for their spontaneous self-assembly. DNA is increasingly being used to organize other nanomaterials in self-assembly processes. Particularly important is that the highly specific interactions between complementary base pairs of DNA can be used to assemble nanomaterials.

The complex organization of biological functions into precise regions of space and time is essential for the functioning of living systems but detailed understanding of the way in which small molecules self-assemble to form complex structures is still fragmentary. Many interesting surface-bound molecular assemblies and phenomena have been revealed by the STM.

Self-assembly is the result of weak non-covalent interactions. This enables error correction during the growth process, thus facilitating long-range order and virtually defect free domains. On the other side, it also results in structures that lack thermal, chemical and mechanical stability for many applications.
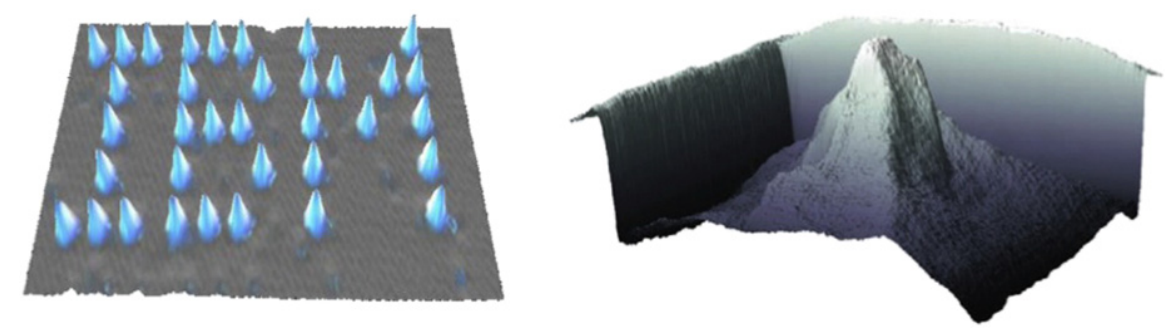

Fig. 2. Micromanipulation in 1989 and 2009. Left: IBM image constructed from Xe atoms in 1989; right: image of the Swiss Matterhorn mountain constructed by optical and electron beam lithography in 2009. Scale is 1.5 billion:1, feature sizes are height: $20 \mathrm{~nm}$, other dimensions: $20 \mu \mathrm{m}$ (www.pcworld.com/article/194787/article.html). 


\section{Nanotechnological products}

Table 1 shows the size range of nanotechnology in the framework of a number of other size dependent phenomena. The table shows dimensions of a few typical nanostructures. We can compare these dimensional aspects of nanotechnology - with its size range between $1 \mathrm{~nm}$ and $100 \mathrm{~nm}$ - with the dimension of a number of other objects: a C-atom is $0.16 \mathrm{~nm}$; a single walled nanotube is $2 \mathrm{~nm}$; the DNA separation of plane of adjacent base pairs is $0.34 \mathrm{~nm}$ and the diameter of the DNA helix $2 \mathrm{~nm}$, a red blood cell is something as $8 \mu \mathrm{m}$, a human hair 50-100 $\mu \mathrm{m}$. Catalysis depends on surface topology in the range of 1-10 nm, immunology depends on molecular recognition at $1-10 \mathrm{~nm}$.

The table also shows the range of materials used from metals to insulators, simple inorganic salts and complex organic molecules, even DNA with its immense potential for self-assembly starts playing a prominent role.

Fig. 3 shows the size range of nanotechnology in the framework of a number of other size dependent phenomena in analytical chemistry and spectroscopy that will be further elaborated on in this paper.

There is an enormous and quickly increasing range of applications and functional uses. Most of the present applications of nanotechnology are based on passive structures (nanoparticles, nanotubes, nanocomposites, nanocoatings...). In the coming decade active nanostructures will increasingly appear (electronics, sensors, targeted drugs...), while further away beyond 2020, third generation nanotechnology will be based on guided molecular assembly, 3-D networking, robotics, and further away (around 2040) molecules by design and evolutionary structures [6].

\subsection{Two examples}

Important aspects of nanomaterials depend on either their composition/structure or on their size. One important set of examples of nanoscopic materials based on structure are those based on carbon and its different allotropes; another group is based on size, with nanoparticles.

\subsubsection{Carbon allotropes}

When an element exists in several structural forms, it is said to exhibit allotropy; the individual forms are called allotropes. The most current naturally occurring examples are carbon black or coal, graphite (one of the softest materials) and diamond, the hardest material.

Carbon is the element that provides the basis for life but is also important for many technological applications, including drugs and numerous chemicals. It is the basis of the chemical industry.

Modern allotropes of carbon are featuring actors in the nanorevolution. Buckminsterfullerene was discovered in 1985 (Kroto, Smalley and Curl, Nobel Prize in Chemistry 1996). It consists of a spherical, ellipsoid, or cylindrical arrangement of a few dozen of carbon atoms. Fullerenes were named after Richard Buckminster Fuller, the architect who designed the geodesic dome that resembles spherical fullerenes in their appearance. Spherical fullerenes looks like a soccer ball, and are also called "buckyballs" whereas cylindrical fullerenes are identified as "buckytubes" or "nanotubes." Fullerenes are often called carbon- 60 because it is the most widely studied and used, although there are also higher mass fullerenes with different geometric structures, such as, with 70, 76, 78 and 80 carbon atoms. Carbon-60 was the start of the development of a number of carbon allotropes that went on with the study of nanotubes (invention of carbon nanotubes,1991, Iijima) and graphene - known since long but first isolated as individual graphene planes in 2004 (Nobel Prize in 2010, Geim and Novoselov). Fullerenes are similar in structure to graphite and composed of stacked graphene sheets of linked hexagonal rings; but they may also contain pentagonal (or sometimes heptagonal) rings. In 1993, a class of carbon nanotube was discovered, with just a single layer. These single-walled nanotubes are generally narrower than the multi-walled tubes. With diameters typically in the range of $1-2 \mathrm{~nm}$, they tend to be curved rather than straight. Graphene consists of one-atom-thick planar sheet of sp2-bonded carbon atoms that are packed in a honeycomb crystal lattice. Graphite itself consists of many graphene sheets stacked together.

With their outstanding properties and potential applications these synthetic carbon allotropes play an important role in nanoscience and nanotechnology. Many applications illustrate their unique scientific and technological importance. At present, particularly graphene is a fast growing product of nanoscience and technology. Its twodimensional hexagonal lattice of carbon atoms has been found to have remarkable physical and chemical properties, and is also being considered for many diverse applications.

The remarkable properties manifested by nanotubes and graphene arise from their structure as an atomically thin mesh of carbon atoms arranged in a honeycomb hexagonal pattern. The very strong carboncarbon bonds produce an exceptionally high strength-to-weight ratio. As was quoted in the Nobel Prize announcement of the physics prize in 2010, graphene has a breaking strength which is more than 100 times stronger than the strongest steel. An almost invisible hammock made out of graphene could hold a cat without breaking. The hammock would weigh less than one $\mathrm{mg}$, corresponding to the weight of one of the cat's whiskers.

The symmetry of the carbon atom arrangement in the hexagonal lattice also provides low electrical resistance opening up electronics applications. Small variations in carbon structure are able to create diverse new properties. Nanotubes can be made semiconducting or metallic by changing their diameter, length or their twisting angle between the lines of hexagons and the direction of the tube. Also, there are differences between single tubes and those in which several cylinders are nested inside each other in multi-walled nanotubes.

\subsubsection{Nanoparticles}

Examples of an important category of nanomaterials based primarily on size are nano-particles and nano-powders. Clusters are particles with well-defined structure and composition, while nanoparticles are species of less precise composition and size distribution (colloids).

Table 1

Typical dimensions of nanostructures.

Adapted from reference [7].

\begin{tabular}{lll}
\hline Nanostructure & Size $(\mathrm{nm})$ & Material \\
\hline Clusters, nanocrystals, quantum dots & Radius 1-10 & Insulators, semi-conductors, metals magnetic materials \\
Other nanoparticles & Radius 1-10 & Ceramic oxides \\
Nanobiomaterials & Radius 5-10 & Membrane protein \\
Nanowires & Diameter 1-100 & Metals, semiconductors, oxides, sulphides, nitrides \\
Nanotubes & Diameter 1-100 & Carbon, chalcogenides \\
Nanobiorods & Diameter 5 nm & DNA \\
Surfaces \& thin films & Thickness 1-1000 & Insulators, semiconductors, metals, DNA \\
2-D arrays/nanoparticles & Area several nm ${ }^{2}-\mu^{2}$ & Metals, semiconductors, magnetic materials \\
3-D superlattices/nanoparticles & Radius several nm & Metals, semiconductors, magnetic materials \\
\hline
\end{tabular}




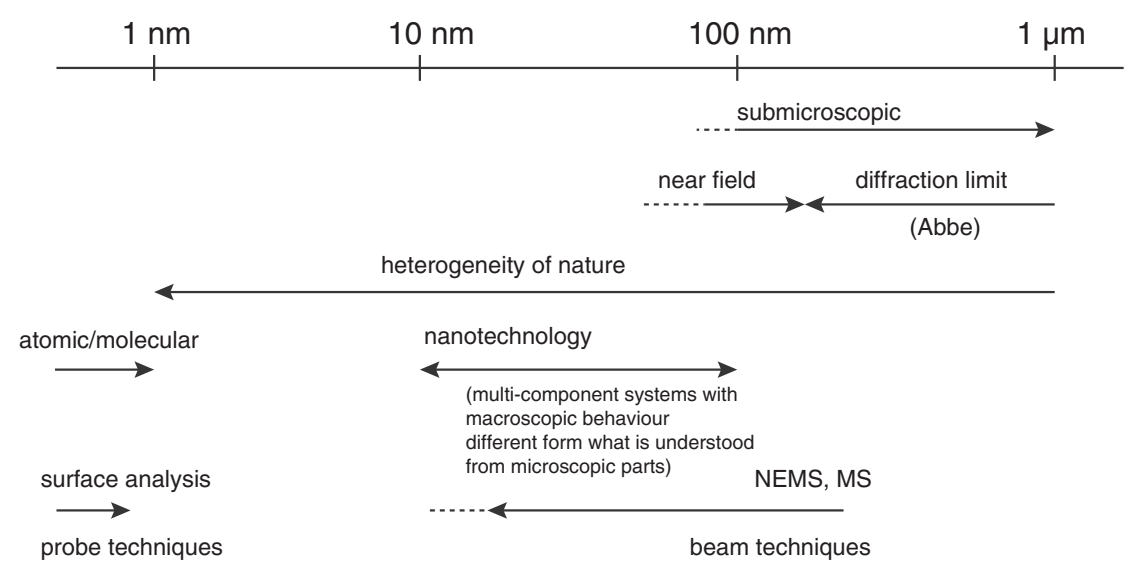

Fig. 3. Dimensional aspects that put nanotechnology in relation with other size dependent phenomena and analytical chemistry and imaging tools.

Nano-powder is a name given to many materials between 1 and $10 \mathrm{~nm}$ with a narrow size distribution. Clusters, nanosize particles and nanopowders have a wide range of simple compositions, carbon, silica, gold, silver, or are more complex as oxides, or semiconducting such as, for instance, quantum nano-dots (QD). QDs are resistant to photobleaching and have a high quantum yield of close to $90 \%$.

Assembling nanoparticles as building blocks into complex functional structures is becoming an important area of research for nanotechnology applications. There is a wide and widening range of applications: they are used in catalytic processes, as coatings, as delivery agents for drugs, and as imaging agents. They are also used as the building blocks for functional structures and complex assemblies. But before we go on with them we turn our attention to nanoscience and the prominent role played in it by analytical chemistry.

\section{Nanoscience and analytical chemistry}

Barriers to the rational design of nanomaterials are the lack of basic knowledge in domains such as physics, chemistry and biology. Observing, correlating and understanding structure and function is a basic requirement for the reproducible production of nanomaterials. If nanoscience and nanotechnology have been connected, but distinctly different endeavors in the past, in the nano realm, they become intimately intertwined and so much connected to become undistinguishable. Increasingly, they are now leading together to newly commercialized and innovative products and future-oriented applications.

Essential for future developments - and this is a major task of nanoscience - is to keep track of the composition and the structure. The analytical capabilities to do this must also move from static measurements to dynamic real-time tools.

These most important aspects of nanoscience as a determining factor in advances are summarized in the two historic quotes. Richard Feynman already emphasized in his well known historic talk at Caltech of 1959 how important it is to be able to "see" things on the atomic level, meaning with this that it is imperative for scientific development that it is necessary to be able to measure the position of atoms with absolute confidence.

" ...but I am not afraid to consider the final question as to whether, ultimately - in the great future - we can arrange the atoms the way we want; the very atoms all the way down."

The second quote,

"If you want to know the function, determine the structure"

comes from Francis Crick in 1989 in his historic account on the discovery of the double helix model of 1953. It underlines the importance of the determination of structure to explain the function of the most basic construction material of life.

\subsection{Analytical chemistry}

There exists, at present, an impressive range of instrumentation encompassing a wide array of continuous wave, frequency domain, time domain and diffuse correlation spectroscopy systems for imaging analysis, covering the electromagnetic spectrum. The variety of experiments in which these methods are applied is diverse and covers compositional, structural and dynamic studies.

At present, practically the entire electromagnetic spectrum can be used for analytical and imaging purposes. Large fractions of the enormous spectral region between the infrared and hard X-rays is prominent in a number of imaging applications. Techniques such as those involving probe analytical techniques give rise to a variety of deexcitation processes involving electrons, ions, neutrals or photons that can be used for the characterization of heterogeneous materials.

Today, atomic scale observation and determination of structure are possible with high accuracy. Scanning probe microscopes (SPM) and high-resolution transmission electron microscope (HRTEM) have enabled direct images of structures at the atomic scale. For instance, atoms can be localized in space with a precision of the order of a few tens of a picometer with the HRTEM. Fig. 4 shows the atomic scale determination of surface facets in gold nanorods [9]. The picture was obtained with the FEI Titan 50-80 transmission electron microscope. This instrument installed in June 2010 in the Electron Microscopy for Materials Sciences (EMAT, University of Antwerp) delivers the ultimate performance in TEM and scanning transmission electron microscopy (STEM) available today. Its double aberration correctors offer an amazing $0.05 \mathrm{~nm}$ resolution in TEM and $0.08 \mathrm{~nm}$ in STEM.

\subsubsection{Biomimetics}

The reasons for the need of the determination of composition and structure in nature are numerous. One example is a topic of growing importance, biomimetics - also called bionics or biognosis - derived from the Greek word "biomimesis" and with the meaning of mimicking biology or natural concepts. Nature has gone through natural evolution over several billion years and by trial and error evolved into objects with high performance. Hence, the way nature fabricates and uses nanostructures is considered an inspiration for taking ideas from nature and to exploit them in applications.

One, often quoted example of biomimetics, consists in surface treatments to induce non-wetting, super-hydrophobicity and selfcleaning. Various natural surfaces including the leaves of several plants - for instance the lotus plant - are super-hydrophobic and do not wet. This is due to the presence of a wax coating on a high surface roughness surface structure as revealed by scanning electron 

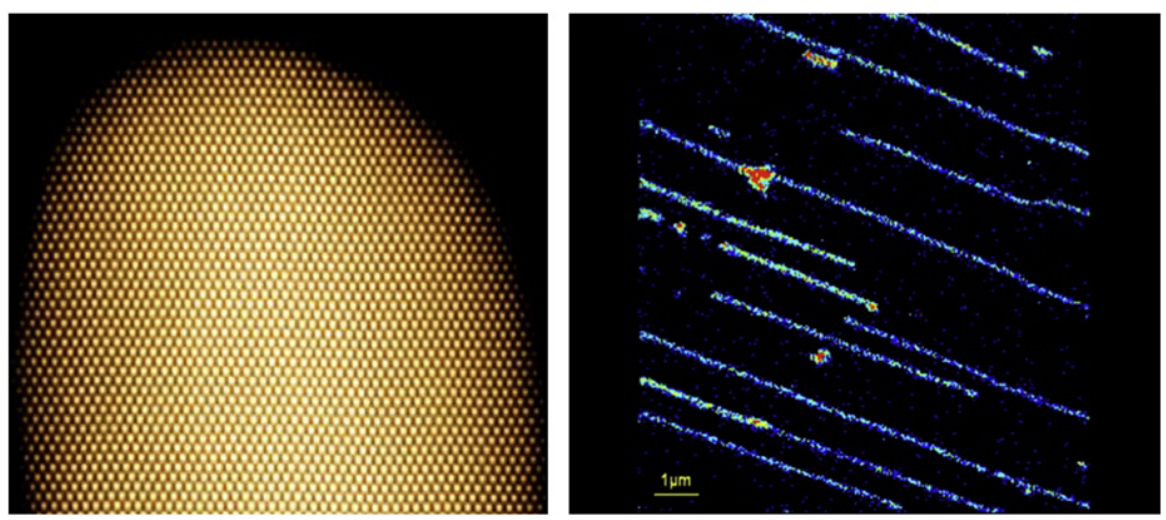

Fig. 4. a) Individual gold atoms in gold nanorods as seen in transmission electron microscopy. Reproduced with permission from ref. [46]. b) Labelled DNA with isotopically modified nucleotides and then aligned on silicon surfaces using the NanoSIMS 50.

Reproduced with permission from Ref. [34].

microscopy. The effect has been called the "Lotus-Effect" and may become of great biological and technological importance. When used in technological applications this effect has various applications, e.g., selfcleaning windows and solar cells, paints, utensils, roof tiles, textiles. It can also reduce drag in fluid flow, e. g. in microscopic or nanochannels.

"How it comes that geckos can climb walls?"

is a question addressed by Andre Geim in his Nobel Lecture in 2010. Careful studies of composition, structure and morphology have revealed that the answer of the question resides with the animal's sticky feet due to submicron size hairy toes. These can now be mimicked with polydimethylsiloxene (PDMS) structures of micron sized dimensions at the surface.

\subsection{Chemical analysis and nanoscience/technology}

The featuring actors in the development of nano-analysis originate with steady developments in analytical chemistry on one side and developments in nano-size materials and tools on the other side. Their combination and interaction led to the possibilities that are now appearing, culminating in the development of sensitive high sensitivity analytical imaging tools with unprecedented spatial discrimination potential. Fig. 3 shows the size range of nanotechnology and different size dependent aspects in analytical chemistry.

\subsubsection{Imaging and analysis}

The acquisition of an optical image is only the first step toward comprehensive data analysis. At present, microscopic techniques accomplish this first step in conjunction with electronic detectors, image processors, and various display devices as integrated extensions of the imaging system. Chemical or structural composition comes then as an additional observation tool. Major analytical techniques for spatially confined analysis and imaging are based on spectroscopic techniques covering various areas of the electromagnetic spectrum or on mass spectrometry derived from methodologies that are summarized under the denominator of "beam analysis". Beam analysis covers most of the spectrometric and mass spectrometric tools for imaging analysis.

The development of spectroscopic techniques capable of compositional mapping on the spatial scale of a few $\mathrm{nm}$ became a topic of increasing interest in recent years. When we need information on local variation of the composition of a material a general experimental approach is based on scattering: we shoot radiation or particles in a well-prepared state on the target and detect radiation or particles coming out of the target as a result of various forms of interactions. In the case of surface analysis the most basic questions we want to solve with this approach are sometimes quite simple such as, for example, whether the surface is clean or which elements are present on the surface or not.

More complex are questions such as which chemical compound are there and what is the exact geometric structure of the surface or what is the coverage and its distribution. In the more general conditions where also lateral dimensions are concerned (for 3-D analysis) questions are related to the elemental or molecular composition and the heterogeneities in composition. Is it random or is it structured? Most of these techniques achieve this by making use of intense particle or X-ray beams and provide essential information down to the atomic scale with a sensitivity and specificity that is impossible to reach by conventional techniques.

Chemical imaging methods reveal the chemical composition on a spatially resolved level over a range of length scales ( $\mathrm{mm}$ to $\mathrm{nm}$ ). They make use of a variety of excitation mechanisms and detection schemes (raster scanning, multichannel detection, parallel channel detection). Most imaging methods are based on one-dimensional (point, 1-D) observations that can be systematically moved over a given surface to produce two-dimensional (2-D) images. While it is sometimes possible to derive 3-D information from such images through stereological considerations, it is often desirable to obtain direct 3-D information on the structure and composition of a particular object. 3-D images can be obtained by collecting 2-D images in slices obtained through sequential sectioning by e.g. the removal of material from the surface with ion beam etching (depth profiling, see further). An accurate full 3-D representation can thus be obtained. The alternative consists in the measurement inside of a given sample by penetrating X-ray, particle or electron beams. Tomographic techniques allow the direct 3-D measurement and analysis of microscopic or nanoscopic objects.

The development of imaging analysis is based on progress in making reliable instrumentation and methodology that responds to scientific and technological needs. Their main concerns in this development are advances in spatial resolution, sensitivity and accuracy. Different methods to reach such goals are often complementary but they all have distinct strengths and weaknesses.

The quote that follows was made by the Committee on Revealing Chemistry through Advanced Chemical Imaging, National Research Council (USA), visualizing chemistry: The Progress and Promise of Advanced Chemical Imaging [7]:

“...sustained efforts are needed to facilitate understanding and manipulation of complex chemical structures and processes. Chemical imaging offers a means by which this can be accomplished by allowing the acquisition of direct, observable information about the nature of these chemistries. By linking technological advances 
in chemical imaging with a science-based approach to using these new capabilities, it is likely that fundamental breakthroughs in our understanding of basic chemical processes in biology, the environment, and human creations will be achieved".

Microscopic and sub-microscopic imaging methods capable of identifying specific atoms and molecules have significantly improved our understanding nature and technological objects. Optical imaging techniques have revolutionized our ability to study the microscopic world. Simple optical microscopy methods have played a large role in scientific fields such as geology and cellular and molecular biology; unfortunately they do not provide sufficient chemical specificity. Imaging methods capable of identifying atoms and specific molecules have significantly improved our understanding processes on the microscopic scale. Tools that provide elemental and molecular information with high spatial resolution are becoming increasingly important for the development of nanotechnology and nanoscience. Analytical techniques that are available for the characterization of the atomic and molecular composition and structure at the bulk level often fail for the analysis of such materials.

\subsubsection{Sensitivity}

Present research explores the extreme limits of analytical chemistry: the characterization of attomole $\left(10^{-15}\right)$ to zeptomole $\left(10^{-18}\right)$ quantities of atoms or molecules because there is the need to detect such small amounts of analyte within nanometer size surfaces or volumes [8]. The primary challenge is to conceive methods and instrumentation for the accurate identification of as little as a few thousand of analyte species (atoms or molecules), or even less. The inherent conflict between sensitivity and spatial resolution is a fundamental trade-off in microanalytical methods, with high spatial resolution for detecting the signals generated being confronted by the needs for improvements in detection sensitivity. Absolute sensitivities at the attogram level become increasingly more common in the literature.

In general, we need to distinguish two different approaches for microanalysis and its systematic pursuit in imaging analysis, the destructive techniques based on sputtering or ablating material and the (except for radiation damage) non-destructive techniques such as those based on the interaction of electrons or X-rays. In principle, in the last group the sensitivity (and the precision) can be increased by using a higher flux of input particles (increasing source brightness) or by counting longer, without sacrificing the spatial resolution. Other factors then start dominating for the correct interpretation of the result, such as inaccuracies in the interpretation of the results e.g. in the interpretation of the background. A second challenge is to convert a measurement into reliable analytical information. Even pushing the limits of technology to the extreme for obtaining a measurement as spectacular as the detection of a single atom or molecule, is often insufficient for solving the problem, for instance when this problem is related to obtain understanding and control of a complex chemical process. Measurements must be related to the physico-chemical system sampled in terms of reliable concentration data and/or spatial but also temporal localization. This is a matter of metrology, but metrological concepts are at present not yet available in this field. Accurate analysis at the microscopic/sub-microscopic level is very important in fundamental scientific research and is of growing importance for the characterization at submicron/nanoscale level in various areas of materials technology. For this reason an accurate metrological approach is needed [9]. At present, few CRMs or RMs suitable for laterally defined analysis have been produced. However, metrologically sound measurements require absolute (primary) methods to link results of chemical measurements to the SI (international system of units) and the development of CRMs at the nanoscale level. Achieving this will be a formidable challenge for the coming years.

In chemical imaging the detection limit is different as a concept than for bulk spectroscopic analysis. In imaging however, each pixel is characterized by a corresponding spectrum in which a specific analyte is either detectable or not and this depends on the homogeneity of the sample and the spatial resolution of the technique used.

\subsubsection{Abbe-Thomson diffraction limit}

Imaging also has limitations in spatial resolution and conventionally cannot go beyond a given limit of lateral resolution, the diffraction limit of light or, more generally, electromagnetic radiation. Diffraction fundamentally limits the resolution that a microscope is able to achieve to ca half the wavelength of the light used.

In his 1959 lecture, Feynman could safely make his prediction since, for instance when an electron is accelerated in a $100 \mathrm{keV} \mathrm{mi-}$ croscope, its wavelength is ca $4 \mathrm{pm}$ which is a distance far shorter than that separating the atoms in a solid or molecule. It took more than 40 years of steady work to measure and correct the various lens aberrations and to iteratively adjust the necessary lens currents in EM for achieving full aberration corrected electron microscopy. $\mathrm{X}$-rays with their wavelength in the range up to $10 \mathrm{~nm}$ are also able to deal with the size range of nanoscience/technology within the limitations of the diffraction limit. It is for the spectral region between the UV and the IR that the diffraction limit became a serious problem for nanoscience/technology.

It is only since the turn of the century - and thanks to the pushing force of nanotechnology and nanoscience (especially in biology) that several approaches of super-resolution microscopy (localization microscopy) were developed [10]. At present these techniques have been optimized to obtain a spatial resolution considerably better than the limit imposed by the wavelength of light, down to a few $\mathrm{nm}$ for visible light. Scanning near-field optical microscopy (SNOM) uses an optical probe with a sub- wavelength-sized opening, within a distance comparable to the aperture's radius, r, to a sample. Progress in the methodology led to the development of "nano-optics" able to manipulate light in sub-wavelength dimensions. Nano-local spectroscopy became a common tool for sub-100 nm spatial resolution optical imaging [11,12].

Near-field techniques are used for scattering, fluorescence, Raman scattering and provide information on single molecules and functional materials down to the $10 \mathrm{~nm}$ level. In addition time-resolved measurements and the study of interactions between molecules became possible. Recent advances in far-field fluorescence microscopy have led to substantial improvements in image resolution with near-molecular resolution of 20-30 nm in two lateral dimensions, imaging at 3-D resolution remaining a challenge [13]. At present a number of different techniques based on the "engineering" of the point spread distribution (PSD), the response envelope on a point radiation source due to diffraction) remediate this problem. Different other approaches also provide sub-diffraction limit spatial resolution, sometimes allowing detection at or near the molecular level.

\subsection{Nanomaterials as analytical tools}

Different uses of nanofibers and nanotubes are shown in Fig. 5. The use of nanomaterials as analytical tools - applying them for analysis is another major goal of analytical nanoscience and nanotechnology, and is also addressed with a number of examples in the literature. In practical terms, nanotechnology-based analytical processes can exploit the nanosize and exceptional properties of structured nanomatter. Nanometer scale analytical systems that are based on nanosized materials, are limited in size and weight and thus scale well as physical size is reduced. Nanotechnological analytical systems, which exploit the exceptional physicochemical properties of nanomaterials, account for most current uses of analytical nanoscience.

Metallic nanoparticles can be used to increase the sensitivity of fluorescent detection because they generate a phenomenon known as metal-enhanced fluorescence increasing fluorescence lifetime and quantum yields [14]. They also result in localized surface plasmon 
resonance (LSPR). Quantum dots have the potential to solve many of the problems associated with organic fluorophores in near infrared spectroscopy. Quantum dots comprise an inorganic core and shell of metal and an outer organic coating. The inorganic core and shell enables tuning of fluorescence with a narrow bandwidth enabling multiplexed detection of molecular targets [15].

The successful integration of such new concepts into analytical nanosystems is at present still at a development stage, but applications are spectacular. By attaching molecules (individual retinal chromophores found in receptor cells) to C60 molecules, it became possible to trap them inside single-walled carbon nanotubes and image them using HR-TEM, thus insuring that individual molecules are well separated and are protected from electron-beam damage during the observation. Multiple molecularly resolved images can be obtained with sub-second time resolution [16]

Nanoscale carbon-based materials, including single walled nanotubes (SWNTs), multiple walled nanotubes (MWNTs) and carbon nanotubes, and fibers can be used in sensing and detection. In particular these materials can be used for applications in sorption and preconcentration of heavy metals ions and organic molecules prior to their detection by spectroscopic, chromatographic and electrochemical techniques. With their ability to incorporate various functional groups, electrospun nanofibers are a good sorbent substrate for solid phase extraction based techniques [17].

\subsubsection{Sensors}

Nanomaterials such as nanoparticles have unique physicochemical properties and they can be used as chemical and biological sensors. Gold nanoparticles (AuNPs) are the most extensively investigated nanomaterials, due to their distinct physical and chemical attributes. It is easy to synthesize stable AuNPs and they have unique, tunable optical properties. With their high surface-to-volume ratio, after appropriate modification processes they offer possibilities for functionalization with ligands for the specific sensing of targets with excellent biocompatibility. AuNPs have become excellent scaffolds for the fabrication of novel sensors, and lots of innovative approaches have developed different techniques for sensing in a rapid, efficient manner [18].

Graphene is receiving significant attention for chemiresistor (CR) and field-effect-transistor (FET) sensor devices. Applications have been demonstrated for the use of chemically derived graphene, as graphene oxide (GO) for CR/FET chemical and biological sensors [19].

Bio-adhesion between silica surfaces and biomolecules is usually done using a chemical linker rather than through direct adsorption.
This provides a stronger adsorption. Nanopatterning and chemical linker method can be used to improve adhesion of biomolecules on silicon surfaces. DNA origami is the nanoscale folding of DNA to create arbitrary two and 3-D shapes [20].

Connecting a patterned silicon substrate with bio-molecules opens new opportunities for assembling nanostructures. Snippets of DNA molecules can be used for making connections between different nano-objects. Organic and biological molecules can be attached to silicon via an intermediary gold layer and a carbon-sulfur-gold bond or via siloxane chemistry directly on oxidized silicon. By building up a scaffolding of multiple molecular layers it is possible to imitate the surface of a living object.

The real impact of nanotechnology appears when the simple structures discussed above are integrated in more complex entities called Micro Electromechanical Systems (MEMS). MEMS are structures obtained through the integration of mechanical elements, sensors, actuators, and electronics on one silicon substrate through successive generations of microfabrication technology. When MEMS are reduced in size by scaling down they arrive in the size domain of nanotechnology and are named Nano Electro Mechaniccal Systems (NEMS). Fig. 6 shows an example of a biomolecule receptor in a field effect transistor (ChemFET), a chemical field-effect transistor as a chemical sensor that can be used to detect atoms, molecules and ions in liquids and gases. While its function is electrochemical not spectroscopic, we nevertheless want to draw attention to these type of structures in this review.

\subsubsection{NEMS}

The small mass density and large mechanical stiffness makes carbonnanotube or graphene based NEMS ideal candidates for ultrasensitive mass detectors that do not require disruptive ionization of the analyzed molecule as in conventional mass spectrometers. Experimental effort has gone into fabricating NEMS employing carbon nanotubes as mechanical resonators [21].

Recent experiments reveal design principles for the construction of ultrasensitive nanotube mass sensors with single yoctogram mass resolution at room temperature while cooling opens the possibility of sub-yoctogram mass resolution [22]. Devices consisting of vibrating sheets of graphene suspended over micron-sized trenches, can also be used as highly sensitive, robust, mass detectors. The performance of monolayer graphene nanomechanical resonators with electrical readout have been described by Chen et al. [23]. The enormous stiffness and low density of graphene coupled to a more favorable surface
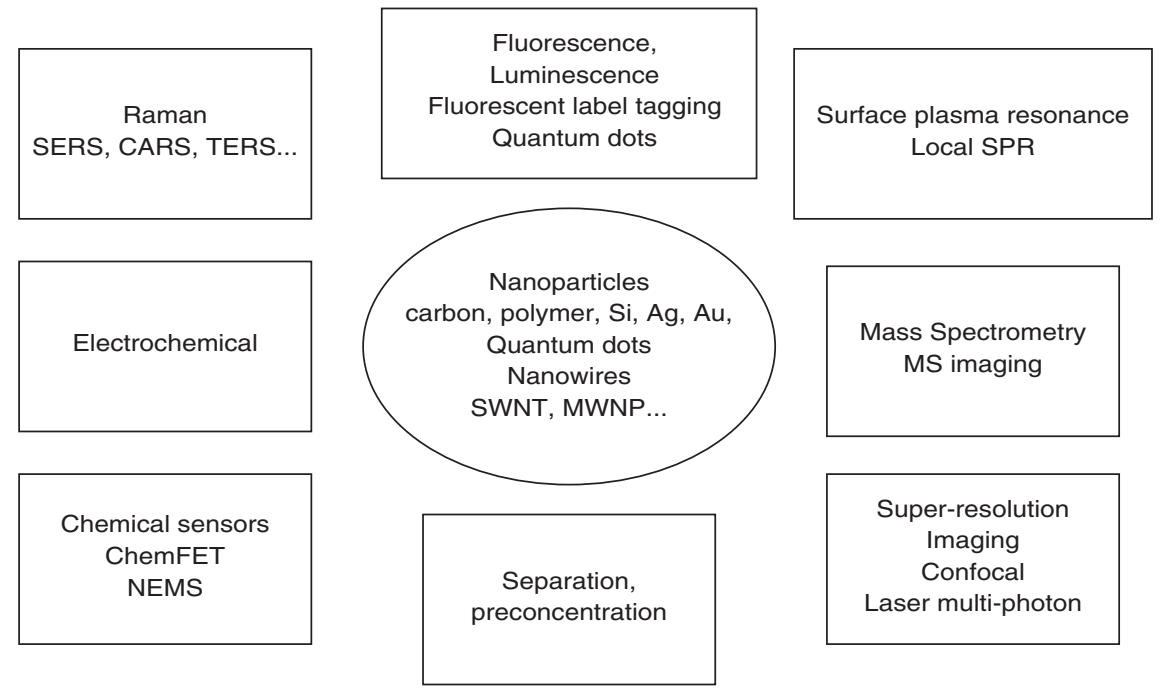

Fig. 5. Different uses of nanofibers and nanotubes in chemical analysis. 


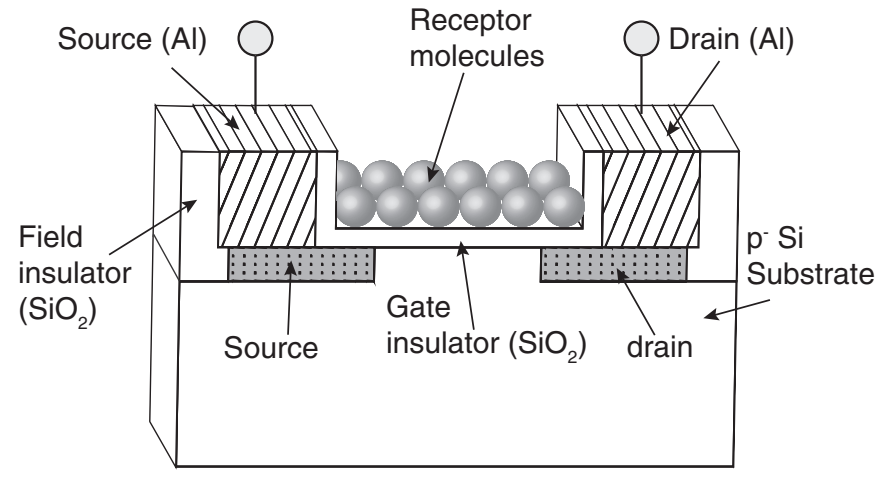

Fig. 6. Integration of biomolecule into a field effect transistor as specific sensor.

configuration for attachment of analytes make it an ideal material for NEMS applications.

\subsubsection{Nanoparticles, quantum dots and spectroscopy}

Nanoparticulate contrast systems such as quantum dots, gold nanoparticles, and dye doped silica nanoparticles offer better photo stability, higher quantum yield, and in vitro and in vivo stability as compared to the conventional contrast dyes. They are used in many in vivo imaging platforms with optical, magnetic resonance imaging (MRI), computed tomography (CT), ultrasound, positron emission tomography (PET), and single photon emission computed tomography (SPECT) [17].

Nanoparticle-based NIR nanoprobes including NIR-emitting semiconductor QDs, lanthanide doped up-converting nanoparticles, and NIR dye-containing nanoparticles are applied for in vivo imaging [24].

Most commercially available QDs have a semiconductor core, often a mixture of cadmium and selenium, measuring about 2-10 nm in diameter. This core is surrounded by a shell, usually of another semiconductor material, and an outer polymer or inorganic layer. In semiconductor quantum dots, the electronic wave functions are squeezed into small areas. Stretching them in a controllable yet simple way profoundly affects their properties and can give them characteristics important for practical applications. The motion of single molecules labeled with fluorescent QDs can be monitored in life cells over time scales ranging between milliseconds to hours in order to determine individual trajectories of molecules with an accuracy at the nm level. Single quantum dot tracking (SQT) thus constitutes an extremely sensitive tool to explore how molecules in cells form assemblies that ensure cellular structures and functions $[25,26]$.

Single molecule spectroscopy and imaging (SMS) for measuring the signal of individual fluorescent labels is of growing interest because it provides information on a single cell instead of the population average of many cells exposing normally hidden heterogeneities [27]. Most of the super-resolution imaging applications involve protein-based labeling of proteins with a fluorescent tag or a dye-labeled antibody. In this way it becomes possible to follow its identity, and modifications in vivo.

Labeling techniques using chemical and biological reactions may cause undesirable changes in the sample, Raman scattering and Coherent anti-Stokes Raman scattering spectroscopy (CARS) allows label-free molecular identification and imaging of macromolecules within the single cell and continues to make major advances but the measurement specificity of protein molecules is limited [28]. Surface enhanced Raman scattering (SERS) and surface enhanced resonance Raman scattering (SERRS) are able to detect substances down to single molecule level but because it is required that metal substrates either have roughened surfaces or take the form of nanoparticles to realize a substantial Raman signal, its applications are limited up to now. Recently, innovative approaches demonstrated that when a layer of gold nanoparticles coated by an ultrathin insulating shell of silica or aluminum is used, considerable Raman signal amplification is realized. Such shell-isolated nanoparticle-enhanced Raman spectroscopy could be used to probe pesticide residues on the surfaces of yeast cells and citrus fruits. Eventually, such techniques could be useful in materials science and the life sciences, as well as for food safety control and the detection of drugs, explosives and environmental pollutants [29].

\subsubsection{Mass spectrometry}

There are several approaches for mass spectrometry based imaging. Imaging mass spectrometry (IMS) has expanded with the advent of new ionization techniques over the years. The development of molecular imaging techniques was driven by uses for the analysis at the single cell level for cells and tissues. Techniques include, amongst others, Matrix-Assisted Laser Desorption Ionization (MALDI) [30]. Lateral resolution in MALDI has been reported to be as good as 0.6$0.7 \mu \mathrm{m}$, but it is more common to consider a lateral resolution on the order of a few $\mu \mathrm{m}$, unsuitable for localized analysis on the sub-micrometer scale) [31]. Imaging mass spectroscopic methods that are able to reach sub-micrometer lateral resolution include static or dynamic Secondary Ion Mass Spectrometry (S-SIMS, D-SIMS), which can attain sub-micrometer imaging resolution [32]. One approach for D-SIMS is based on the use of the NanoSIMS 50, an instrument able to detect and quantify ions under 300 Da with a very high spatial resolution and high mass resolution at high transmission. It is able to yield an isotopic image of the surface with a lateral resolution of around $50 \mathrm{~nm}$ [33]. Using the nanoSIMS 50, Cabin-Flaman et al. [34] were able to detect and image labelled DNA with isotopically modified nucleotides and then aligned on silicon surfaces using the NanoSIMS 50 (Fig. 4b). Isotopically labeled, single DNA fibers, uniformly aligned on this surface, were detected with a resolution of $50 \mathrm{~nm}$ (i.e., 150 base pairs).

Laser ablation ICPMS (LA-ICPMS) has gained widespread popularity for elemental imaging due to its wide multi-element capacity, its large dynamic range, its ultra-trace sensitivity, its potential for isotope analysis and its high spatial resolution, down to $c a 15-50 \mu \mathrm{m}^{2}$. Koch and Günther [35] estimated that fs laser conditions will be able push depth resolution towards the nanosize range but that 3-D analysis on the nanometer scale will only be possible when the laser radiation is delivered through near-field techniques and assistance of the laser by several kinds of positioning tools [36]. SNOM has been developed into a tool for highly localized laser-based imaging analysis using laser ablation mass spectrometry. The scanning probe images surface structures non-invasively and aligns the ion beam to particular sub-microscopic regions of interest. We refer to recent reviews on mass spectrometric imaging for detailed surveys [37-39].

The detection of engineered nanomaterials can be also carried out by using separation techniques coupled with a mass spectrometric detector. As an example, capillary electrophoresis, size exclusion chromatography, hydrodynamic chromatography and field-flow fractionation have been used for the quantification of silver nanoparticles in different materials [40].

\section{Applications}

The range of application areas of nanotechnology include applications in microelectronics for circuits, sensors, displays, data storage materials of all kinds for paints, coatings (including sun-tans), energy applications for solar cells, fuel cells etc., in environmental applications and scientific instrumentation of various kinds, including many concerned with chemical analysis.

The market of nanotechnology products is expanding rapidly. From 147 billion USD in 2007 it is expected to increase to over one trillion in 2015 , a healthy growth rate of almost $30 \%$ per annum! 


\subsection{Applications in biology and medicine}

Biomolecules (DNA, lipids, metabolites...) are essential in the biochemistry of cells. Monitoring the spatial and temporal behavior within single live cells remains difficult but is not impossible. Of special importance are also applications in the biosciences, pharmaceutics and medicine. Biological processes take place at the molecular level with interactions on extremely small size level and this is - also the realm of nanoscience and nanotechnology. Basically analytical biology and biochemistry evolve around the determination of basic molecular properties related to size, weight and position. In addition, there is a complex interplay between molecules in living systems resulting in a continuous alteration of these properties at the molecular level. There is consequently a need for methods that provide insight into these processes to increase understanding of fundamental processes. Opportunities in cell biology and most importantly in medicine in general abound with possibilities for sensors, molecular imaging, early detection of diseases etc.

A significant challenge in translating nanoscale methods into medically or biologically relevant tools results from the disparity in scientific culture separating medical disciplines from the physical sciences. Traditionally, for example, experts in cellular signaling and signal transduction are applying biochemical methods and molecular approaches to address biological problems relevant to health sciences. Based on their background, they may not take immediate advantage of the rapid evolution in chemical, physical, and material sciences facilitating the usage of more sophisticated nanoscale technologies and methods for addressing complex problems within their discipline. In turn, scientists advancing nanotechnology may not be aware of the most pertinent questions and analytical challenges to be addressed in cell physiology or - more generally - in biomedicine.

\section{Nanotoxicology, ecotoxicology}

The interactions between nanomaterials and cells, animals, humans and the environment are complex and much research is necessary to understand in detail how the physical, chemical and other properties of nanomaterials influence these interactions, and thus determine the ultimate impact of nanomaterials on health and the environment. Research during the last twenty years has confirmed that nanoscale materials manifest unexpected toxicity. Their impact on human health and the environment is, at present, quite incompletely understood. There is also an ongoing debate about the regulation of nanomaterials.

One obvious difficulty in nano-toxicology is that materials that are not at all harmful in their bulk form may well be toxic on the nanoscale. Nanoparticles are more likely to react with cells and various biological components such as proteins, and to travel through organisms, which increases their chances of interacting with the organism to induce inflammatory and immunological responses. For example, nanoparticles may acquire a 'corona' of proteins when exposed to biological fluids, and this layer is thought to influence the way the cell interacts with a nanoparticle.

In Europe, the regulations for registration, evaluation, authorization and restriction of chemicals, in short the REACH regulations are in force since 2007. New European cosmetics regulations require ingredients that contain nanomaterials to be listed on product labels by 2013. In the United States, the US Food and Drug Administration issued draft guidance to help producers to determine whether their products utilize nanomaterials while the Environmental protection Agency has issued draft guidelines for nanomaterials.

On the subject of nanoparticles the European Commission issued a draft definition in 2011 that stipulates:

"...a material that consists of particles with one or more external dimensions in the size range $1 \mathrm{~nm}-100 \mathrm{~nm}$ for more than $1 \%$ of their number";and/or has internal or surface structures in one or more dimensions in the size range $1 \mathrm{~nm}-100 \mathrm{~nm}$; and/or has a specific surface area by volume greater than $60 \mathrm{~m}$ per cubic $\mathrm{cm}^{3}$, excluding materials consisting of particles with a size lower than 1 nm..."

We can conclude on all this that distinctions are necessary between real risks and perceptual risks, and between both of these and regulations. Regulations with no scientific justification could effectively do more harm than good. The quantification of risks in coherent way is the key to future. It is needless to say that in line with the increasing market size there is both in the USA and Europe, a steeply increasing environmental, health and safety (EHS) budget with the purpose to understand and address potential risks to health and environment.

It will be important in the coming years to understand how physical and chemical properties of nanomaterials govern their interactions and responses. Chemical analysis needs to play an important role in this work. The detailed characterization of the materials is also essential in all areas of nanotoxicology.

\section{Conclusions}

Nanotechnology is now well-established, it is applied to many unrelated products and technologies. Its use in science and technology includes improvements of existing technology but also opens up exciting new opportunities. By achieving ultimate precision manufacturing every atom in the right place - nanotechnology complex and intricate structures and can do this easily and inexpensively, reduces manufacturing costs and providing savings of raw materials and energy.

In addition we see a very intimate link between nanoscience and nanotechnology on one side and emerging strategies for development. The previous remarks show also the close link with nature provided by biomimetics, mimicking biology or natural concepts, as an example. Fig. 7 shows as an example a false-colored scanning electron microscopy image of hybrid protein-inorganic nanoflowers [41]. As such, it illustrates the heuristic way (using experience-based techniques for problem solving and discovery) in which the presently available visualization and analysis tools are able to explore, understand and apply for applications, structure and form in nature as it evolved.

For chemical analysis on the nanolevel a number of spectrometric tools are used to complement the information available through physical techniques such as atomic force microscopy, scanning tunnelling microscopy and electron microscopy. These techniques are mostly based on the use of spectrometric tools such as Raman spectroscopy, near-field microscopy, laser ablation mass spectrometry and nanooptical sensing. Beam methods of analysis based on ion laser of X-ray beams, are being optimized at the nanoscale level and now operate at the nanoscale resolution for 2-D and 3-D analyses [10,42].

Many published examples show how solutions were found for both of the spatial limitations posed by the diffraction limit and the specificity in chemical detection at the reduced dimensions level. Super-resolution microscopy is one of the most significant developments in imaging since the invention of the microscope. Its capabilities developed since only just a decade ago, but now extend the boundaries of 2-D and 3-D imaging [43].

The instrumental development efforts should give rise to significant advances of the analytical methodologies at two distinct levels [44]:

- Improvements in resolving characteristics well into the sub-100 nm range laterally and with a depth resolution below the nm level, while preserving or optimizing sensitivity. The lateral and depth resolution of the analytical instrument are the focal point for the analysis of nanoscale structures. Their determination requires reference materials with well-defined features in the size range between the dimensions of crystal lattices $(<1 \mathrm{~nm})$ and $\sim 150 \mathrm{~nm}$. 


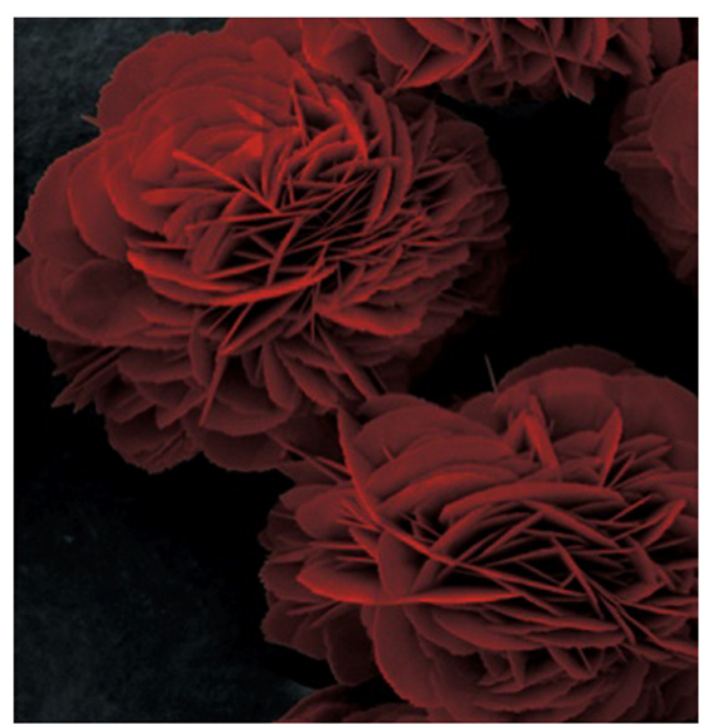

Fig. 7. False-colored scanning electron microscopy image of hybrid protein-inorganic nanoflowers.

Reproduced with permission from Ref. [41].

- Advances in accuracy and the study of systematic error sources. This should lead to quantitative/traceable results and systematic errors with measurement uncertainty that can be reproduced in laboratories using the same techniques. This implies establishing a measurement traceability scheme for key-methods in each instrument class. The experimental methods discussed above often deliver images that require subjective judgment. Background effects and the errors in their estimation are frequently underestimated. In order to make measurements more objective and quantitatively traceable, tools for image analysis adhering to the concepts of modern metrology must be developed.

\section{References}

[1] B. Bhushan, Springer Handbook of Nanotechnology, 3rd Revised and Extended Edition Ed, Springer, Berlin, 2010.

[2] F. Mercuri, M. Baldoni, A. Sgamellotti, Towards nano-organic chemistry: perspectives for a bottom-up approach to the synthesis of low-dimensional carbon nanostructures, Nanoscale 4 (2012) 369-379.

[3] D.A. Buck, K.R. Shoulders, An approach to microminiature printed systems, Papers and Discussions Presented at the December 3-5, 1958, Eastern Joint Computer Conference: Modern Computers: Objectives, Designs, Applications, ACM, 1958, pp. 55-59.

[4] K.K. Berggren, Lithography, Nanoscale 3 (2011) 2662-2662.

[5] S. Lemon, IDG News Service, IBM Creates 1:5 Billion Scale Model of the Matterhorn, http://www.pcworld.com/article/194787/article.html2010.

[6] M.C. Roco, Nanoscale science and engineering: unifying and transforming tools, AlChE J. 50 (2004) 890-897.

[7] Committee on Revealing Chemistry through Advanced Chemical Imaging National Research Council USA Board on Chemical Sciences, Visualizing Chemistry: The Progress and Promise of Advanced Chemical Imaging, National Academy Press, Washington, D.C., 2006

[8] F. Adams, From nano to yocto, and beyond? Talanta 85 (2011) 1230-1232.

[9] R.K. Leach, R. Boyd, T. Burke, H.U. Danzebrink, K. Dirscherl, T. Dziomba, M. Gee, L. Koenders, V. Morazzani, A. Pidduck, D. Roy, W.E.S. Unger, Y. Yacoot, The European nanometrology landscape, Nanotechnology 22 (2011).

[10] F. Adams, C. Barbante, History and present status of imaging analysis, Talanta 102 (2012) 16-25.

[11] D. Richards, Z. Anatoly, Preface, Philos. Trans. R. Soc. Lond. A Math. Phys. Eng. Sci 362 (2004) 699-700.

[12] V.T. Yadugiri, R. Malhotra, 'Plenty of room' - fifty years after the Feynman lecture, Curr. Sci. 99 (2010) 900-907.

[13] B. Huang, W.Q. Wang, M. Bates, X.W. Zhuang, Three-dimensional super-resolution imaging by stochastic optical reconstruction microscopy, Science 319 (2008) $810-813$.

[14] H. Li, C.Y. Chen, X. Wei, W.B. Qiang, Z.H. Li, Q. Cheng, D.K. Xu, Highly sensitive detection of proteins based on metal-enhanced fluorescence with novel silver nanostructures, Anal. Chem. 84 (2012) 8656-8662.
[15] A.M. Smith, X.H. Gao, S.M. Nie, Quantum dot nanocrystals for in vivo molecular and cellular imaging, Photochem. Photobiol. 80 (2004) 377-385.

[16] Z. Liu, K. Yanagi, K. Suenaga, H. Kataura, S. Iijima, Imaging the dynamic behaviour of individual retinal chromophores confined inside carbon nanotubes, Nat. Nanotechnol. 2 (2007) 422-425.

[17] S. Chigome, G. Darko, N. Torto, Electrospun nanofibers as sorbent material for solid phase extraction, Analyst 136 (2011) 2879-2889.

[18] Q. Xiao, H. Gao, C. Lu, Q. Yuan, Gold nanoparticle-based optical probes for sensing aminothiols, TrAC Trends Anal. Chem. 40 (2012) 64-76.

[19] R.K. Paul, S. Badhulika, N.M. Saucedo, A. Mulchandani, Graphene nanomesh as highly sensitive chemiresistor gas sensor, Anal. Chem. 84 (2012) 8171-8178.

[20] P.W.K. Rothemund, Folding DNA to create nanoscale shapes and patterns, Nature 440 (2006) 297-302.

[21] B. Lassagne, Y. Tarakanov, J. Kinaret, D. Garcia-Sanchez, A. Bachtold, Coupling mechanics to charge transport in carbon nanotube mechanical resonators, Science 325 (2009) 1107-1110.

[22] J. Lischner, T.A. Arias, Material limitations of carbon-nanotube inertial balances: possibility of intrinsic yoctogram mass resolution at room temperature, Phys. Rev. B 81 (2010) 233409.

[23] C.Y. Chen, S. Rosenblatt, K.I. Bolotin, W. Kalb, P. Kim, I. Kymissis, H.L. Stormer, T.F. Heinz, J. Hone, Performance of monolayer graphene nanomechanical resonators with electrical readout, Nat. Nanotechnol. 4 (2009) 861-867.

[24] P. Pantazis, J. Maloney, D. Wu, S.E. Fraser, Second harmonic generating (SHG) nanoprobes for in vivo imaging, Proc. Natl. Acad. Sci. U. S. A. 107 (2010) 14535-14540.

[25] F. Pinaud, S. Clarke, A. Sittner, M. Dahan, Probing cellular events, one quantum dot at a time, Nat. Methods 7 (2010) 275-285.

[26] M. Baker, Nanotechnology imaging probes: smaller and more stable, Nat. Methods 7 (2010) 957-962.

[27] S.J. Lord, H.L.D. Lee, W.E. Moerner, Single-molecule spectroscopy and imaging of biomolecules in living cells, Anal. Chem. 82 (2010) 2192-2203.

[28] K. Fujita, N.I. Smith, Label-free molecular imaging of living cells, Mol. Cells 26 (2008) 530-535.

[29] J.F. Li, Y.F. Huang, Y. Ding, Z.L. Yang, S.B. Li, X.S. Zhou, F.R. Fan, W. Zhang, Z.Y. Zhou, D.Y. Wu, B. Ren, Z.L. Wang, Z.Q. Tian, Shell-isolated nanoparticle-enhanced Raman spectroscopy, Nature 464 (2010) 392-395.

[30] M. Setou, N. Kurabe, Mass microscopy: high-resolution imaging mass spectrometry, J. Electron Microsc. 60 (2011) 47-56.

[31] K.J. Boggio, E. Obasuyi, K. Sugino, S.B. Nelson, N.Y.R. Agar, J.N. Agar, Recent advances in single-cell MALDI mass spectrometry imaging and potential clinical impact, Expert Rev. Proteomics 8 (2011) 591-604.

[32] M. Senoner, W.E.S. Unger, SIMS imaging of the nanoworld: applications in science and technology, J. Anal. At. Spectrom. 27 (2012) 1050-1068.

[33] S.G. Boxer, M.L. Kraft, P.K. Weber, Advances in imaging secondary ion mass spectrometry for biological samples, Annual Review of Biophysics, 2009, pp. 53-74.

[34] A. Cabin-Flaman, A.F. Monnier, Y. Coffinier, J.N. Audinot, D. Gibouin, T. Wirtz, R. Boukherroub, H.N. Migeon, A. Bensimon, L. Janniere, C. Ripoll, V. Norris, Combed single DNA molecules imaged by secondary ion mass spectrometry, Anal. Chem. 83 (2011) 6940-6947.

[35] J. Koch, D. Gunther, Review of the state-of-the-art of laser ablation inductively coupled plasma mass spectrometry, Appl. Spectrosc. 65 (2011) 155A-162A.

[36] M.V.Zoriy, J.S. Becker, Near-field laser ablation inductively coupled plasma mass spectrometry: a novel elemental analytical technique at the nanometer scale, Rapid Commun. Mass Spectrom. 23 (2009) 23-30.

[37] L.A. McDonnell, R.M.A. Heeren, Imaging mass spectrometry, Mass Spectrom. Rev. 26 (2007) 606-643.

[38] J.S. Fletcher, N.P. Lockyer, J.C. Vickerman, Developments in molecular SIMS depth profiling and 3d imaging of biological systems using polyatomic primary ions, Mass Spectrom. Rev. 30 (2011) 142-174.

[39] R. Lobinski, C. Moulin, R. Ortega, Imaging and speciation of trace elements in biological environment, Biochimie 88 (2006) 1591-1604.

[40] M.E. Hoque, K. Khosravi, K. Newman, C.D. Metcalfe, Detection and characterization of silver nanoparticles in aqueous matrices using asymmetric-flow field flow fractionation with inductively coupled plasma mass spectrometry, J. Chromatogr. A 1233 (2012) 109-115.

[41] J. Ge, J. Lei, R.N. Zare, Protein-inorganic hybrid nanoflowers, Nat. Nanotechnol. 7 (2012) 428-432.

[42] F. Adams, Analytical atomic spectrometry and imaging: looking backward from 2020 to 1975, Spectrochim. Acta Part B 63 (2008) 738-745.

[43] G. Shtengel, J.A. Galbraith, C.G. Galbraith, J. Lippincott-Schwartz, J.M. Gillette, S. Manley, R. Sougrat, C.M. Waterman, P. Kanchanawong, M.W. Davidson, R.D. Fetter, H.F. Hess, Interferometric fluorescent super-resolution microscopy resolves 3D cellular ultrastructure, Proc. Natl. Acad. Sci. U. S. A. 106 (2009) 3125-3130

[44] F. Adams, L. Van Vaeck, R. Barrett, Advanced analytical techniques: platform for nanomaterials science, Spectrochim. Acta Part B 60 (2005) 13-26.

[45] Y. Ju-Nam, J.R. Lead, Manufactured nanoparticles: an overview of their chemistry, interactions and potential environmental implications, Sci. Total. Environ. 400 (2008) 396-414.

[46] B. Goris, S. Bals, W. Van den Broek, E. Carbó-Argibay, S. Gómez-Graña, L.M. Liz-Marzán, G. Van Tendeloo, Atomic-scale determination of surface facets in gold nanorods, Nat. Mater. 11 (2012) 930-935. 